\title{
Preliminary Observations on the Antihypertension Action of Dabigatran Etexilate in Patients With Nonvalvular Atrial Fibrillation: A Prospective Multicenter Single-Arm Interventional Study
}

\author{
Tomohiko Yamamoto ${ }^{\mathrm{a}, \mathrm{b}}$, Hidenori Urata ${ }^{\mathrm{a}}$
}

\begin{abstract}
Background: In Japan, the number of patients with atrial fibrillation continues to grow with the aging of the population. Prevention of cardiogenic cerebral embolism is extremely important in patients with atrial fibrillation. While warfarin has long played a major role for this purpose, a new oral anticoagulant, dabigatran etexilate (dabigatran), has demonstrated superior efficacy and safety in recent years. We conducted a multicenter prospective interventional study to examine whether dabigatran could demonstrate superiority over warfarin in practical clinical situation.
\end{abstract}

Methods: Among outpatients attending Fukuoka University Chikushi Hospital or clinics registered with the Chikushi Cardiovascular Disease Clinical Research Network (Chikushi-JRN), 143 patients with nonvalvular atrial fibrillation (NVAF) were enrolled in this study and followed up for 12 months after initiation of dabigatran therapy. The primary endpoint was occurrence of cerebral embolism or systemic embolism, while secondary endpoints were: 1) Bleeding events; 2) Changes in the activated partial thromboplastin time (aPTT); 3) Adverse events; and 4) Changes in blood pressure and pulse rate.

Results: During the follow-up period, none of the patients developed cerebral or systemic embolism (the primary endpoint). In addition, there were no bleeding events or other adverse events (the secondary endpoints). aPTT remained stable throughout the 12-month observation period. A significant decrease in systolic blood pressure was observed at 1 month after initiation of dabigatran therapy, and blood pressure was reduced up to 12 months. Blood pressure showed a significant decrease in patients with paroxysmal atrial fibrillation, but not in patients with chronic atrial fibrillation.

Conclusions: Dabigatran showed a stable anticoagulant effect, and its

Manuscript submitted December 18, 2019, accepted January 6, 2020

aDepartment of Cardiovascular Diseases, Fukuoka University Chikushi Hospital, 1-1-1 Zokumyouin, Chikushino, Fukuoka 818-8502, Japan

${ }^{b}$ Corresponding Author: Tomohiko Yamamoto, Department of Cardiovascular Diseases, Fukuoka University Chikushi Hospital, 1-1-1 Zokumyouin, Chikushino, Fukuoka 818-8502, Japan. Email: tomohiko0326@gmail.com safety was confirmed. Dabigatran also reduced blood pressure, which may help to explain why it causes fewer major bleeding events than warfarin.

Keywords: Dabigatran; Hypertension; Antihypertensive; Nonvalvular atrial fibrillation; Oral anticoagulant

\section{Introduction}

The prevalence of atrial fibrillation increases with age. Accordingly, the number of patients with atrial fibrillation continues to grow in Japan with aging of the population, and is expected to exceed one million by 2020 . Although atrial fibrillation is not a lethal arrhythmia, it is the primary risk factor for cardiogenic cerebral embolism and thus requires adequate management. The results of a phase 3 international collaborative study of dabigatran (Randomised Evaluation of LongTerm Anticoagulation Therapy (RE-LY study)), a pioneering novel oral anticoagulant, were reported at the 2009 European Society of Cardiology Congress, demonstrating superior efficacy and safety of dabigatran over warfarin when used to prevent cardiogenic cerebral embolism in patients with nonvalvular atrial fibrillation (NVAF). In the present study, we evaluated the preventive effect of dabigatran on cardiogenic cerebral embolism and the risk of bleeding in patients with NVAF in practical clinical situation. Based on the results of the RE-LY trial that dabigatran is safer than warfarin, we examined clinical factors that may contribute to the reduction in bleeding rate, and it has become clear that blood pressure reduction may have been linked to dabigatran's safety. We investigated the possibility that dabigatran improves various factors related to bleeding safety.

\section{Materials and Methods}

\section{Subjects}

The subjects were NVAF patients aged $<85$ years who were 
initiating or continuing outpatient anticoagulant therapy at Fukuoka University Chikushi Hospital or at clinics registered with the Chikushi Cardiovascular Clinical Research Network (Chikushi-JRN). The study protocol was approved by the ethics committee of Fukuoka University Chikushi Hospital (R12022), and all subjects gave written informed consent to participation.

\section{Exclusion criteria}

Exclusion criteria were as follows: 1) Patients with a history of hypersensitivity to dabigatran; 2) Patients with advanced renal dysfunction, including those on maintenance dialysis (creatinine clearance $<30 \mathrm{~mL} / \mathrm{min}$ ); 3) Patients with hemorrhagic symptoms, a bleeding diathesis, or a hemostatic disorder; 4) Patients with liver dysfunction (serum alanine aminotransferase (AST) and aspartate aminotransferase (ALT) $\geq 2$ times the reference value); 5) Patients with disorders causing a clinically problematic bleeding risk (including hemorrhagic stroke within 6 months before enrollment); 6) Patients with an indwelling spinal or epidural catheter and those in whom the catheter was removed within $1 \mathrm{~h}$ before enrollment; 7) Patients receiving itraconazole; 8) Patients aged $\geq 85$ years; 9) Patients who were pregnant or possibly pregnant; and 10) Patients who were deemed to be inappropriate for the study by the attending physician for other reasons.

\section{Study design}

This was a multicenter prospective interventional study. Patients were registered from September 2012 through October 2014 and were followed up for 12 months from the time of giving consent. In patients switching from warfarin to dabigatran, administration of dabigatran was initiated immediately if the prothrombin time-international normalized ratio (PT-INR) was $<2.0$. If the PT-INR was $\geq 2.0$, dabigatran was initiated after confirming a decrease below 2.0 following discontinuation of warfarin. NVAF patients who were newly starting anticoagulant therapy were immediately commenced on dabigatran. The standard dose of dabigatran was $300 \mathrm{mg} /$ day, which was reduced to $220 \mathrm{mg}$ /day for patients aged $\geq 70$ years or those with moderate renal dysfunction (creatinine clearance: $30-50 \mathrm{~mL} /$ min). Measurement of blood pressure and pulse rate, as well as blood biochemistry tests, were carried out at $0,1,3,6,9$, and 12 months after initiation of treatment. The blood pressure measurement method adopted the average value measured three times in sitting position in the outpatient clinic of each facility.

\section{Clinical outcome}

The primary endpoint was the occurrence of cerebral embolism or systemic embolism. Secondary endpoints were: 1) Bleeding events; 2) Changes in the activated partial thromboplastin time (aPTT); 3) Adverse events; and 4) Changes in blood pressure
Table 1. Background Factors of the Patients

\begin{tabular}{ll}
\hline Age, years & $73 \pm 10$ \\
Male, n (\%) & $98(69)$ \\
BMI, kg/m² & $24.0 \pm 3.1$ \\
HTN, n (\%) & $119(83)$ \\
DM, n (\%) & $33(23)$ \\
CVD, n (\%) & $11(8)$ \\
Pre-warfarin, n (\%) & $59(41)$ \\
PT-INR & $1.47 \pm 0.32$ \\
Drugs & $63(44)$ \\
\multicolumn{1}{c}{ ACE-I/ARB, n (\%) } & $26(18)$ \\
\multicolumn{1}{c}{ CCB, n (\%) } & $16(11)$ \\
\hline
\end{tabular}

Data are shown as the mean \pm SD or $\mathrm{n}(\%)$. BMI: body mass index; HTN: hypertension; DM: diabetes mellitus type 2; CVD: cardiovascular disease; PT-INR: prothrombin time-international normalized ratio; ACE-I/ARB: angiotensin converting enzyme inhibitor/angiotensin receptor blocker; CCB: calcium channel blocker; SD: standard deviation.

and pulse rate.

\section{Statistical analysis}

Statistical analysis was performed by using SAS 9.3 (SAS Institute Inc., Cary, NC, USA). Results are reported as the mean \pm standard deviation (SD). Categorical variables were compared between groups by Chi-square analysis or Fisher's exact test. The differences in continuous variables between groups were examined by Student's $t$-test or Wilcoxon's rank-sum test. Differences were considered to be statistically significant when the $\mathrm{P}$ values were $<0.05$.

\section{Results}

Table 1 summarizes the characteristics of the 143 patients enrolled in the study. Their mean age was 73 years, $69 \%$ were male, and the mean body mass index (BMI) was $24.0 \mathrm{~kg} / \mathrm{m}^{2}$. The prevalence of hypertension (HTN), type 2 diabetes mellitus (DM), and ischemic heart disease (IHD) was $83 \%, 23 \%$, and $8 \%$, respectively. Warfarin was being used by $41 \%$ of the patients at the time of enrollment and their mean PT-INR was 1.47 .

Table 2 lists the changesin antihypertensive medication. There were no changes in antihypertensive medications during the study period in $59 \%$ of patients. The antihypertensive medications were increased in $15 \%$ of patients and unknown in $17 \%$.

Table 3 lists the main laboratory test results for all patients at 0 and 12 months. No significant differences were observed between the two times of assessment, except for high-density lipoprotein cholesterol (HDL-C), low-density lipoprotein cholesterol (LDL-C), and brain natriuretic peptide (BNP). 
Table 2. List of Patients Who Changed Antihypertensive Medication

\begin{tabular}{ll}
\hline No change, $\mathrm{n}(\%)$ & $85(59)$ \\
ACE-I/ARB added, n (\%) & $6(4)$ \\
CCB added, n (\%) & $10(7)$ \\
Diuretics added, n (\%) & $6(4)$ \\
ACE-I to ARB, n (\%) & $4(3)$ \\
CCB to ARB, n (\%) & $2(1)$ \\
ACE-I/ARB reduced, n (\%) & $2(1)$ \\
CCB reduced, n (\%) & $2(1)$ \\
Unknown, n (\%) & $26(17)$ \\
\hline
\end{tabular}

Data are shown as $\mathrm{n}(\%)$. ACE-I/ARB: angiotensin converting enzyme inhibitor/angiotensin receptor blocker; CCB: calcium channel blocker.

\section{Primary endpoint}

None of the patients developed cerebral embolism or systemic embolism (the primary endpoint) during the follow-up period.

\section{Secondary endpoints}

\section{Blood pressure and aPTT in all patients}

Figure 1a shows the changes in aPTT in 90 patients who underwent measurement. Compared with before initiation of dabigatran therapy, significant prolongation of aPTT was observed stably throughout the 12 -month treatment period $(\mathrm{P}<$ $0.001)$. None of the patients developed abnormal prolongation of aPTT.

Figure $1 \mathrm{~b}(\mathrm{~A})$ displays the changes in blood pressure over 12 months in all patients. There was a significant decrease in systolic blood pressure (SBP) at 1 month after initiation of dabigatran therapy $(\mathrm{P}<0.05)$, and it remained significantly lower than the baseline value after 3,6, and 9 months. Although the diastolic blood pressure (DBP) was not significantly reduced at 1 month after initiation of dabigatran, a decreasing trend was observed over time and a significant decrease was noted at 9 months $(\mathrm{P}<0.01)$.

\section{Blood pressure in the switching group and new treatment group}

We compared the changes in blood pressure and heart rate (HR) after dividing the patients into a group switching to dabigatran from warfarin (switching group) and a group newly starting anticoagulant therapy with dabigatran (new treatment group) (Fig. 1b (B)). In the switching group $(\mathrm{n}=58)$, a significant decrease in SBP was observed at 1 month after switching $(\mathrm{P}<0.05)$. Although SBP subsequently remained below the baseline level, the decrease was not significant from 3 months onward. There was no significant decrease in DBP or HR at any time.

In the new treatment group $(n=83)$, SBP showed a de-
Table 3. Changes in Laboratory Values Over 12 Months

\begin{tabular}{llll}
\hline & 0 month & 12 months & P value \\
\hline $\mathrm{Hb}, \mathrm{g} / \mathrm{dL}$ & $13.9 \pm 1.7$ & $13.8 \pm 1.9$ & 0.30 \\
$\mathrm{AST}, \mathrm{IU} / \mathrm{L}$ & $27.2 \pm 10.9$ & $25.3 \pm 9.2$ & 0.18 \\
$\mathrm{ALT}, \mathrm{IU} / \mathrm{L}$ & $24.6 \pm 19.0$ & $20.0 \pm 10.1$ & 0.05 \\
$\mathrm{BUN}, \mathrm{mg} / \mathrm{dL}$ & $16.6 \pm 4.3$ & $16.7 \pm 4.3$ & 0.44 \\
$\mathrm{Cr}, \mathrm{mg} / \mathrm{dL}$ & $0.89 \pm 0.21$ & $0.92 \pm 0.22$ & 0.12 \\
$\mathrm{~T}-\mathrm{Chol}, \mathrm{mg} / \mathrm{dL}$ & $185.0 \pm 33.3$ & $174.8 \pm 31.9$ & 0.05 \\
$\mathrm{LDL}, \mathrm{mg} / \mathrm{dL}$ & $106.1 \pm 29.9$ & $95.4 \pm 26.9$ & 0.02 \\
$\mathrm{HDL}, \mathrm{mg} / \mathrm{dL}$ & $56.8 \pm 13.7$ & $60.9 \pm 14.2$ & 0.04 \\
$\mathrm{TG}, \mathrm{mg} / \mathrm{dL}$ & $133.8 \pm 79.3$ & $116.0 \pm 67.2$ & 0.08 \\
$\mathrm{UA}, \mathrm{mg} / \mathrm{dL}$ & $5.8 \pm 1.3$ & $5.6 \pm 1.4$ & 0.22 \\
$\mathrm{Na}, \mathrm{mEq} / \mathrm{L}$ & $141.3 \pm 2.4$ & $141.2 \pm 2.1$ & 0.38 \\
$\mathrm{~K}, \mathrm{mEq} / \mathrm{L}$ & $4.2 \pm 0.4$ & $4.3 \pm 0.5$ & 0.08 \\
$\mathrm{Cl}, \mathrm{mEq} / \mathrm{L}$ & $104.6 \pm 2.9$ & $104.8 \pm 2.5$ & 0.29 \\
$\mathrm{BG}, \mathrm{mg} / \mathrm{dL}$ & $121.7 \pm 40.2$ & $115.8 \pm 108.3$ & 0.28 \\
$\mathrm{HbA} 1 \mathrm{c}, \%$ & $6.3 \pm 1.0$ & $6.2 \pm 0.6$ & 0.46 \\
$\mathrm{BNP}, \mathrm{pg} / \mathrm{dL}$ & $177.8 \pm 114.5$ & $88.4 \pm 90.5$ & 0.03 \\
\hline
\end{tabular}

Data are shown as the mean $\pm \mathrm{SD}$. Hb: hemoglobin; AST: aspartate transaminase; ALT: alanine aminotransferase; BUN: blood urea nitrogen; Cr: creatinine; T-Chol: total cholesterol; LDL: low density lipoprotein-cholesterol; HDL: high density lipoprotein-cholesterol; TG: triglyceride; UA: uric acid; Na: sodium; K: potassium; Cl: chloride ion; BG: blood glucose; HbA1c: hemoglobin A1c; BNP: brain natriuretic peptide; SD: standard deviation.

crease from 1 month onward and the decline was significant at 6 and 9 months $(\mathrm{P}<0.05)$. DBP also showed a decrease from 1 month onward, and the decrease was significant at 9 months $(\mathrm{P}<0.01)$. HR was significantly decreased from 1 month until the end of the study $(\mathrm{P}<0.01)$ (Fig. $1 \mathrm{~b}(\mathrm{C}))$.

Figure $2 \mathrm{a}, \mathrm{b}$ show SBP and HR data stratified by the presence or absence of switching antihypertensive agents. In the switching group, 17 patients also switched their antihypertensive therapy, whereas it was not changed in 41 patients. There were no changes in SBP or HR in both subgroups. In the new treatment group, 15 patients switched antihypertensive agents and 44 patients remained on the same antihypertensive therapy. In the patients who switched antihypertensive agents, a significant decrease in SBP was observed after 6 and 9 months $(\mathrm{P}<0.05)$, as well as a significant decrease in HR at 12 months $(\mathrm{P}<0.05)$. Although there were no significant changes in SBP and HR in the patients who remained on the same therapy, both parameters showed a decreasing trend. There were no significant changes in DBP in either subgroup.

Changes in blood pressure and HR in the new treatment group without switching of antihypertensive agents stratified by chronic or paroxysmal atrial fibrillation

Figure 2c displays the changes in SBP and HR in patients from the new treatment group who did not switch antihypertensive 

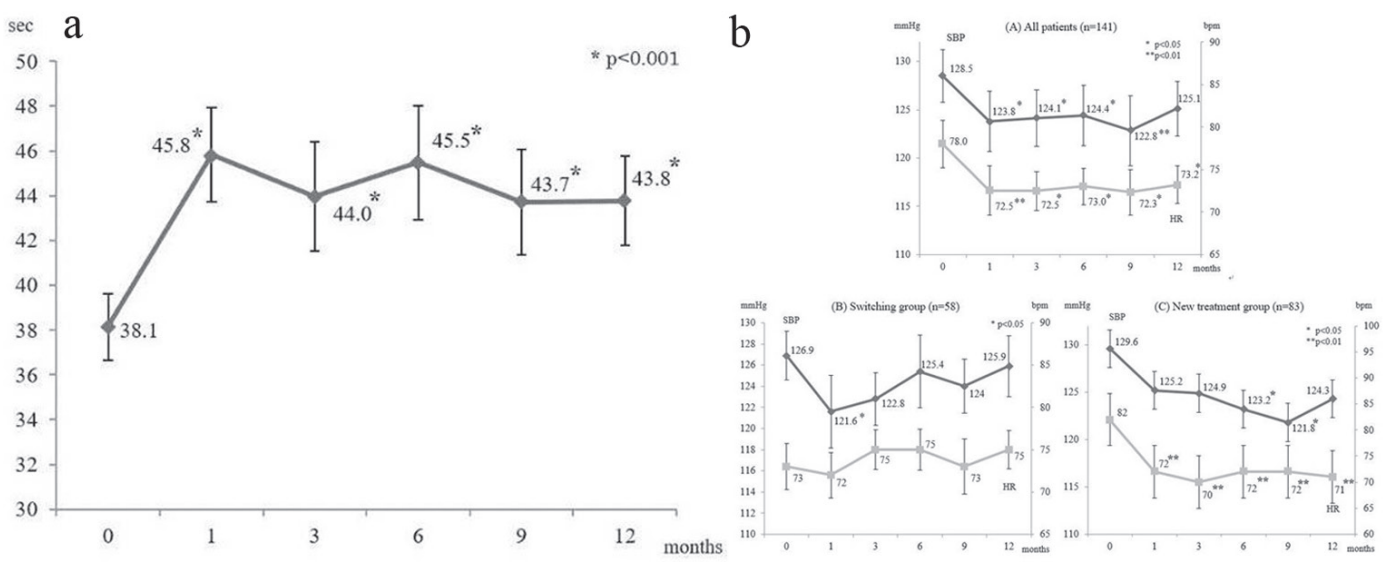

Figure 1. (a) Changes in aPTT; data are shown as the mean \pm SD. (b) Changes in SBP and HR; data are shown as the mean \pm SD. SBP: systolic blood pressure; HR: heart rate; bpm: beats per minute; SD: standard deviation.

agents stratified by the type of atrial fibrillation (chronic or paroxysmal). Fifteen patients were confirmed to have chronic atrial fibrillation and 12 patients had paroxysmal atrial fibrillation. There were no changes in SBP or HR in the subgroup with chronic atrial fibrillation. On the other hand, both SBP and HR decreased in the subgroup with paroxysmal atrial fibrillation and the decline of SBP was significant at 6 months onwards $(\mathrm{P}<0.05)$, while HR was significantly decreased at 3 , 6 , and 12 months $(\mathrm{P}<0.05)$.

No bleeding events or other adverse events were noted in any of the subjects.

\section{Discussion}

Cardiogenic cerebral embolism is one of major events that can cause rapid deterioration of activities of daily living (ADLs), leading to a bed-ridden state, so preventing cardiogenic cerebral embolism by effective anticoagulant therapy is extremely important. HTN is the most common complication in patients with atrial fibrillation [1] and its management is also important, since HTN is involved in the development of atrial fibrillation and increases the risk of cardiogenic embolism.

Structural remodeling (fibrosis) of the atrium contributes to the onset and persistence of atrial fibrillation [2]. Various cardiovascular factors, such as HTN and IHD, combine with non-cardiovascular factors like aging and oxidative stress to promote atrial fibrosis and progression to chronic atrial fibrillation [3].

In normal vessels, thrombin improves vascular endothelial function by promoting the production of nitric oxide and prostacyclin. In contrast, thrombin promotes the production of several cytokines and chemokines in pathological vessels, causing vascular hyperpermeability [4]. As a result, thrombin decreases the bioavailability of nitric oxide by increasing oxidative stress, as well as inducing vascular endothelial dysfunction and plaque growth/destabilization [5, 6]. Moreover, thrombin stimulates the proliferation of vascular smooth muscle cells via the thrombin receptor, which is related to progression of atherosclerosis [7], and it has been suggested that inhibition of thrombin might directly modulate vascular inflammatory cells [8]. In ApoE-deficient mice fed a high-fat diet for 4 weeks, progression of atherosclerosis was compared between groups with or without dabigatran administration, revealing that aortic inflammation and progression of atherosclerosis were suppressed in the mice receiving dabigatran. Based on these findings, it is possible that the antioxidant and anti-inflammatory effects of thrombin inhibition by dabigatran might suppress the progression of atherosclerosis [9].

These findings also suggest that the antioxidant and antiinflammatory effects of dabigatran may inhibit progression of atrial fibrosis in patients with paroxysmal atrial fibrillation, in whom atrial fibrosis is still ongoing.

In post hoc analysis, the group of patients with paroxysmal atrial fibrillation whose antihypertensive therapy was unchanged during the study period demonstrated a significant decrease in blood pressure after initiation of dabigatran, suggesting that the depressor effect of dabigatran was independent of changes in antihypertensive medication.

In our real-world clinical study, it was also suggested that dabigatran could reduce the sympathetic nervous system activity, because HR did not increase despite significant blood pressure reduction.

Generally, decreased sympathetic nervous system activity suppresses inflammatory cytokine production from macrophages and results in anti-inflammatory effect [10]. These mechanisms might be in accordance with our results. Thrombin has been reported to induce inflammation by inducing cytokines and chemokines in vessels, suggesting that the direct thrombin inhibition of dabigatran exerts its anti-inflammatory effect.

Because there have been no reports about a direct effect of dabigatran on blood pressure, it seems likely that the improvement of blood pressure was attributable to its anti-inflammatory and antioxidant effects and suppression of fibrosis. Such clinical findings have not been reported previously and our novel results may offer a new insight into the benefits of dabigatran. Since HTN is an important comorbidity associated with atrial fibrillation, our finding that dabigatran improves the blood pressure as well as prevents cardiogenic cerebral embo- 


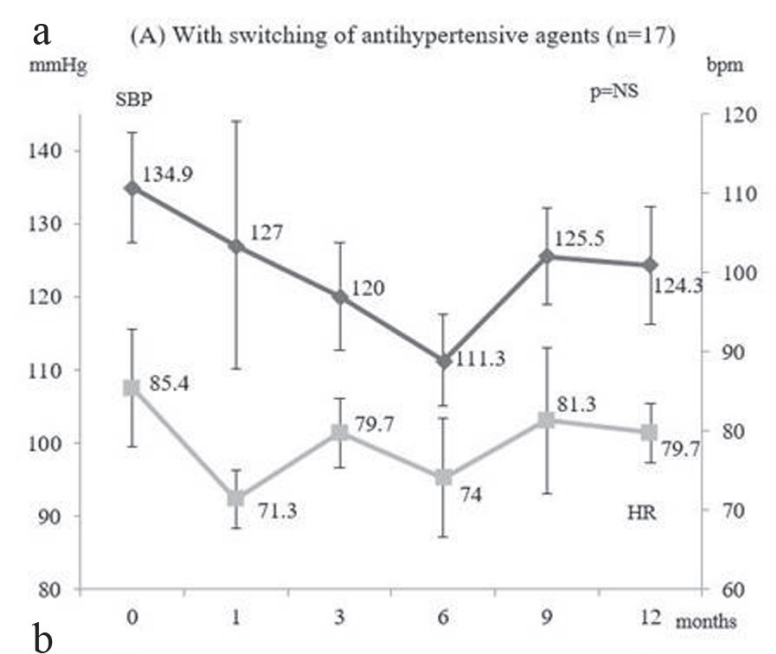

(B) Without switching of antihypertensive agents $(n=41)$
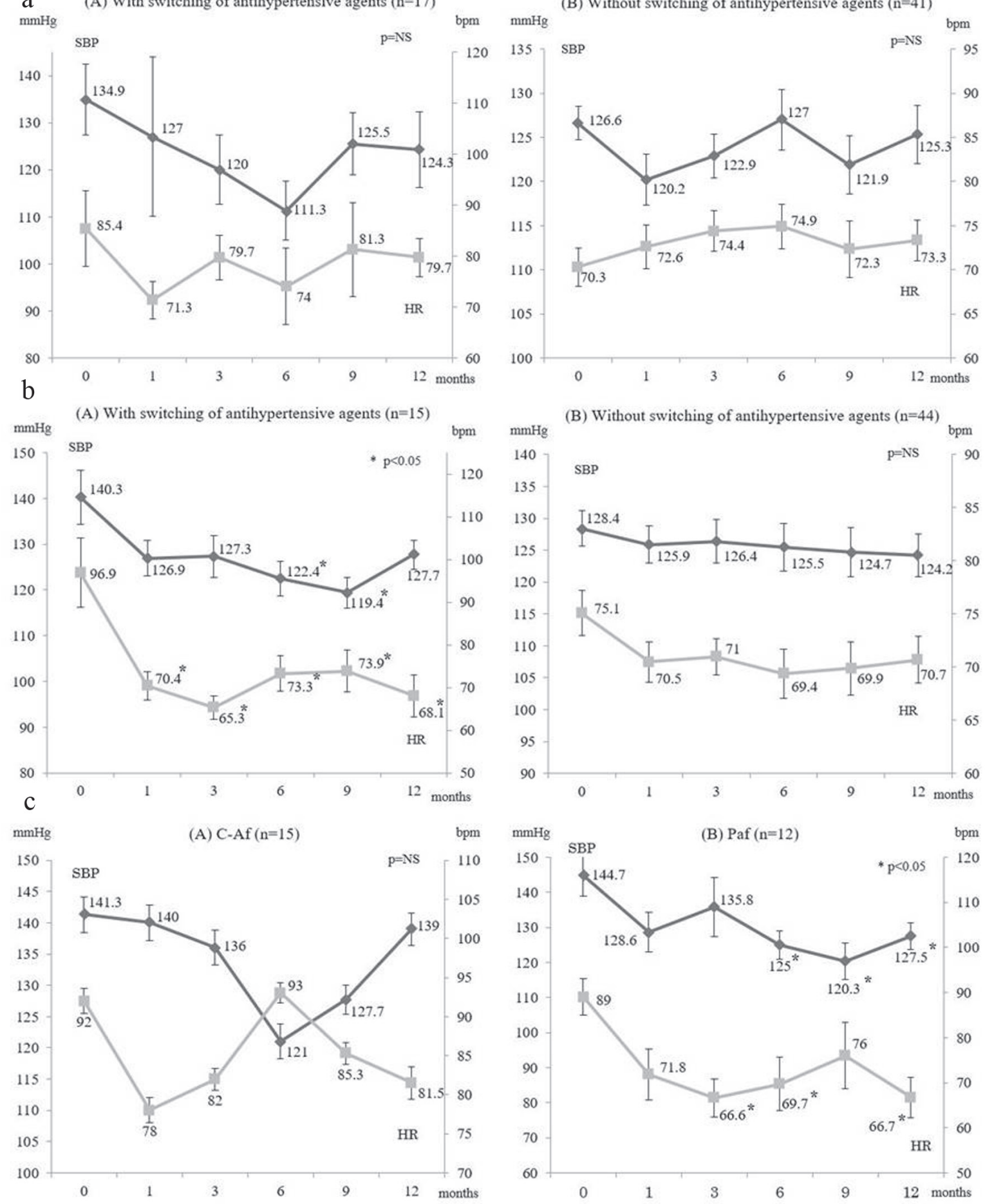

Figure 2. (a) Changes in SBP and HR in the switching group. (b) Changes in SBP and HR in the new treatment group. (c) Changes in SBP and HR in the new treatment group without switching of antihypertensive agents stratified by type of fibrillation. SBP: systolic blood pressure: HR: heart rate; bpm: beats per minute; C-Af: chronic atrial fibrillation; Paf: paroxysmal atrial fibrillation.

lism could be important with regard to selection of medications.

During the 12-month treatment period, none of the patients developed cerebral embolism or systemic embolism, confirming the safety of dabigatran as described in previous reports.

Although it was reported that adherence to dabigatran is poor in patients with NVAF [11], the mean aPTT showed sta- 
ble prolongation throughout the present study, suggesting that our patients showed good adherence. This finding supports the favorable adherence to dabigatran therapy and good safety profile revealed by a post-marketing survey in Japan [12].

With regard to the decrease in blood pressure, another possibility is that improvement of adherence due to participation in this study might have resulted in the Hawthorne effect, which means that results are improved because subjects undergoing an intervention respond to expectations by modifying their behavior. The Hawthorne effect is a known source of bias in research and its possible influence on study results has been pointed out [13]. While the present study was a multicenter investigation, a single-arm design was employed. This means that the observed improvements of HDL-C, LDL-C, and BNP (Table 3) might be related to improved adherence to medication due to the Hawthorne effect. Accordingly, it will be important to confirm the hypotensive effect of dabigatran observed in this study by performing a prospective comparative study in the future.

Despite its single-arm design, this study is the first to demonstrate an antihypertensive effect of dabigatran. However, a two-group comparative study should be conducted in the future to verify our results.

\section{Limitations}

Since the blood pressure variation is larger in patients with atrial fibrillation, it was necessary to set equal conditions for blood pressure measurement. The number of patients in whom we could diagnose chronic or paroxysmal NVAF was very small, but a statistically significant antihypertensive effect was only observed in paroxysmal NVAF group.

Therefore, a two-group comparative study with a larger sample size will be required to confirm the present results.

This clinical study was a single arm study, the primary outcome was safety of dabigatran, and the potential antihypertensive effect of dabigatran was a secondary outcome.

It is necessary to compare dabigatran with placebo and other oral anticoagulants; thus, a prospective study is needed in the future with the antihypertensive effect of dabigatran as the primary endpoint.

\section{Conclusions}

Dabigatran shows a stable anticoagulant effect and its safety is confirmed. We also found that dabigatran reduced the blood pressure, possibly by suppressing vascular and atrial fibrosis. To our knowledge, this is the first report about improvement of blood pressure by dabigatran.

\section{Acknowledgments}

The authors would like to acknowledge the reviewers for their helpful comments on this paper. We thank members of Chikushi-JRN for recruiting patients to the study.

\section{Financial Disclosure}

None to declare.

\section{Conflict of Interest}

We declare that all the authors have read and approved the final version of this. The authors have received financial support from Nippon Boehringer lngelheim Co., Ltd and the ethics and protocol of the present study were approved by the institutional ethics committee (R12-011).

\section{Informed Consent}

All subjects gave written informed consent to participation.

\section{Author Contributions}

TY and HU contributed to the design and implementation of the research, to the analysis of the results and to the writing of the manuscript.

\section{References}

1. Psaty BM, Manolio TA, Kuller LH, Kronmal RA, Cushman M, Fried LP, White R, et al. Incidence of and risk factors for atrial fibrillation in older adults. Circulation. 1997;96(7):2455-2461.

2. Nattel S, Dobrev D. The multidimensional role of calcium in atrial fibrillation pathophysiology: mechanistic insights and therapeutic opportunities. Eur Heart J. 2012;33(15):1870-1877.

3. Nattel S, Opie LH. Controversies in atrial fibrillation. Lancet. 2006;367(9506):262-272.

4. Jawien J, Olszanecki R, Nowak B, Marcinkiewicz J. Apolipoprotein E knockout mice: an experimental model to study inflammatory mechanisms of atherosclerosis and to screen a putative anti-atherogenic properties of drugs. Central European Journal of Immunology. 2012;37(1):3644.

5. Rudolph V, Rudolph TK, Schopfer FJ, Bonacci G, Lau D, Szocs K, Klinke A, et al. Bivalirudin decreases NO bioavailability by vascular immobilization of myeloperoxidase. J Pharmacol Exp Ther. 2008;327(2):324-331.

6. Harrison D, Griendling KK, Landmesser U, Hornig B, Drexler $\mathrm{H}$. Role of oxidative stress in atherosclerosis. Am J Cardiol. 2003;91(3A):7A-11A.

7. Maruyama I, Shigeta K, Miyahara H, Nakajima T, Shin H, Ide S, Kitajima I. Thrombin activates NF-kappa $\mathrm{B}$ through thrombin receptor and results in proliferation of vascular smooth muscle cells: role of thrombin in atherosclerosis and restenosis. Ann N Y Acad Sci. 1997;811:429-436.

8. Martorell L, Martinez-Gonzalez J, Rodriguez C, Gentile 
M, Calvayrac O, Badimon L. Thrombin and proteaseactivated receptors (PARs) in atherothrombosis. Thromb Haemost. 2008;99(2):305-315.

9. Pingel S, Tiyerili V, Mueller J, Werner N, Nickenig G, Mueller C. Thrombin inhibition by dabigatran attenuates atherosclerosis in ApoE deficient mice. Arch Med Sci. 2014;10(1):154-160.

10. Rosas-Ballina M, Olofsson PS, Ochani M, Valdes-Ferrer SI, Levine YA, Reardon C, Tusche MW, et al. Acetylcholine-synthesizing $\mathrm{T}$ cells relay neural signals in a vagus nerve circuit. Science. 2011;334(6052):98-101.

11. Brown JD, Shewale AR, Talbert JC. Adherence to rivaroxaban, dabigatran, and apixaban for stroke prevention for newly diagnosed and treatment-naive atrial fibrillation patients: an update using 2013-2014 data. J Manag Care Spec Pharm. 2017;23(9):958-967.

12. Inoue H, Uchiyama $\mathrm{S}$, Atarashi H, Okumura $\mathrm{K}$, Koretsune Y, Yasaka M, Yamashita T, et al. Post-marketing surveillance on the long-term use of dabigatran in Japanese patients with nonvalvular atrial fibrillation: Preliminary report of the J-dabigatran surveillance. J Arrhythm. 2016;32(2):145-150.

13. McCambridge J, Witton J, Elbourne DR. Systematic review of the Hawthorne effect: new concepts are needed to study research participation effects. J Clin Epidemiol. 2014;67(3):267-277. 